\title{
Organisation syntaxique des constituants autour de l'infinitif dans les textes injonctifs : Étude de cas ${ }^{1}$
}

\author{
Khodabocus, Nooreeda \\ Centre de Recherches en Linguistique Française GRAMMATICA, EA 4521 - Université d'Artois \\ nooreeda@hotmail.com
}

\begin{abstract}
Résumé. Nous proposons un travail sur l'organisation syntaxique des constituants autour d'un infinitif injonctif. En effet, l'utilisation de l'infinitif dans les injonctions nous a amené à nous interroger sur le rapport entre la tournure de la phrase et la nature du texte en question. Dans cette optique, nous proposons une étude de cas sur un corpus constitué de trois différents types de textes utilisant l'infinitif injonctif : des recettes de cuisine (Davies, 2003, Collectif, 2011), un manuel d'entretien et de maintenance des appareils de laboratoire (Organisation mondiale de la Santé, 2008) et un référentiel de compétences de secouristes (Direction de la Défense et de la Sécurité Civiles, 2007). Ces textes s'adressent à des publics différents et ont une visée propre. Dans un premier temps, nous présentons brièvement les caractéristiques linguistiques $\mathrm{du}$ texte injonctif en considérant les différents aspects morphosyntaxique, sémantique, pragmatique, énonciatif, la notion de sujet et les propriétés de l'infinitif injonctif comme forme verbale. Dans un second temps, nous faisons une étude sur corpus. Après avoir expliqué le choix du corpus et la construction de la base de données, nous procédons à une analyse des occurrences recueillies. Nous relevons les différentes structures syntaxiques autour de l'infinitif et nous les examinons en considérant la nature des textes où elles apparaissent. Enfin, nous soumettons une interprétation des résultats obtenus.
\end{abstract}

\begin{abstract}
This paper focuses on the syntactic structure of the injunctive sentences using the French infinitive. Studying the use of the infinitive verb in injunctive texts leads to questioning the link between sentence structure and text type. In this view, we provide a case study based on three text types using the injunctive infinitive, which have different target groups (cooking recipes, a service and maintenance manual and a competency framework for rescuers). We present the properties of injunctive texts from different linguistic perspectives (morphosyntax, semantics, pragmatics, enunciation). We also consider the subject of the injunctive text as well as the characteristics of the infinitive in this context. The collected data will allow us to compare the sentence structures for the different text types.
\end{abstract}




\section{Introduction}

Dans cet article, nous nous intéresserons à l'utilisation de l'infinitif dans le texte injonctif, et plus précisément, à l'organisation syntaxique des constituants autour de cette forme verbale. En effet, l'emploi de l'infinitif dans les injonctions nous a amenée à nous interroger sur le rapport entre la tournure de la phrase et la nature du texte en question, le texte injonctif permettant des constructions qui semblent être propres à ce genre de discours (élision de l'objet direct, emploi absolu qui ne se retrouve pas dans les formes finies, sauf à l'impératif, etc.). Dans cette optique, nous proposons une étude de cas sur un corpus constitué de trois différents types de textes utilisant l'infinitif injonctif : des recettes de cuisine (Davies, 2003; Collectif, 2011), un manuel d'entretien et de maintenance des appareils de laboratoire (Organisation mondiale de la Santé, 2008) et un référentiel de compétences de secouristes (Direction de la Défense et de la Sécurité Civiles, 2007). Ces textes s'adressent à des publics différents et ont une visée propre.

Dans un premier temps, nous présenterons brièvement les caractéristiques linguistiques du texte injonctif en considérant les différents aspects morphosyntaxique, sémantique, pragmatique, énonciatif, la notion de sujet et les propriétés de l'infinitif injonctif comme forme verbale.

Dans un second temps, nous ferons une étude sur corpus. Après avoir expliqué le choix du corpus et la construction de la base de données, nous procéderons à une analyse des occurrences recueillies. Nous relèverons les différentes structures syntaxiques autour de l'infinitif et les examinerons en considérant la nature des textes où elles apparaissent. Nous traiterons aussi du comportement particulier des circonstants dans ce contexte. Enfin, nous soumettrons une interprétation des résultats obtenus.

\section{Caractéristiques linguistiques du texte injonctif}

\subsection{Morphosyntaxe du type injonctif}

La phrase injonctive peut se construire de différentes manières. Lorsque le sujet est une deuxième personne, l'injonction s'exprime le plus souvent au moyen de l'impératif. Pour les autres personnes, on trouve le subjonctif :

(1) Va dans ta chambre !

(2) Battez les œufs en neige.

(3) Qu'il vienne me voir avant de quitter le bureau !

À l'écrit, on rencontre également l'utilisation de l'infinitif injonctif :

(4) Battre les œufs en neige. 
On peut aussi trouver des constructions autour de :

- Verbes illocutoires directifs (Je t'interdis de sortir après 19h.) ;

- $\quad$ Le présent gnomique (Le couteau se tient dans la main droite. ${ }^{2}$ ) ;

- Le présent à valeur injonctive (Tu fais tes devoirs tout de suite!) ;

- $\quad$ Le futur (Tu achèteras du pain en rentrant!) ;

- Les verbes modaux devoir et pouvoir (Vous devez rendre ce devoir pour la semaine prochaine.) ;

- $\quad$ La périphrase « il faut que » (Il faut que la viande soit bien dorée.).

Le discours injonctif s'adresse généralement à un interlocuteur. Le sujet renvoie à un pronom personnel désignant un humain. Dans les constructions métaphoriques, on peut rencontrer un sujet humanisé. S'il est possible d'adresser à un chien un énoncé tel que :

(5) Arrête d'aboyer!

c'est uniquement dans la mesure où l'animal est « humanisé » et qu'on lui parle comme on parlerait à une personne. On peut aussi rapprocher cet emploi à un souhait du locuteur, ce qui donne dans ce cas plutôt un optatif.

Cette contrainte sur l'interprétation du sujet est d'autant plus forte pour l'infinitif injonctif que cette forme n'existe qu'à l'écrit en français contemporain.

À l'impératif et à l'infinitif, le SN sujet est effacé. Au niveau morphosyntaxique, on retrouve néanmoins une trace du sujet dans la désinence verbale à l'impératif, alors qu'à l'infinitif, la place de sujet est vide syntaxiquement, et cette forme verbale ne porte pas non plus de trace morphologique de la personne :

(6) Mange cette pomme !

(7) Tournez à droite après l'église.

(8) Appliquer sur le visage humide. ${ }^{3}$

Cependant, on peut trouver des énoncés à l'infinitif où il y a une référence à une deuxième personne, ce qui indique que le sujet existe, bien qu'absent en surface :

(9) Mouiller votre visage à l'eau chaude. ${ }^{4}$

\subsection{Aspects sémantiques}

$\mathrm{Au}$ niveau sémantique, l'injonction fait partie d'un type de modalité puisqu'il y est question de «l'attitude du locuteur par rapport au contenu propositionnel de son énoncé » (Le Querler, 1996: 14). Afin de situer l'injonction dans cette typologie, nous prenons appui sur le classement proposé par Gosselin (2010), qui a l'avantage d'être très détaillé et de bien faire la distinction entre ce qui relève de la subjectivité et de l'objectivité d'une part, et de ce qui est d'ordre idéologique et d'ordre institutionnel d'autre part.

Gosselin (2010) propose des paramètres constitutionnels à propos de la modalité. Ces paramètres se divisent en trois groupes (p. 59): les paramètres conceptuels qui «permettent de définir un 'concept modal' $[\ldots]$ indépendamment de son instanciation dans un énoncé particulier » (instance de validation, 
direction d'ajustement monde-énoncé, force de validation), les paramètres fonctionnels qui « précisent le mode de fonctionnement du concept modal de l'énoncé » (niveaux syntaxique, logique et énonciatif) et enfin un 'métaparamètre' «qui indique par quelle voie les valeurs des autres paramètres ont été calculés ». Ces paramètres permettent ainsi de voir les distinctions entre les différentes modalités. Nous retenons donc le classement proposé qui comprend les modalités aléthiques, épistémiques, appréciatives, axiologiques, bouliques et déontiques (cf. Gosselin, $2010: 309$ ).

Pour des contraintes évidentes d'espace, nous ne pouvons, ici, passer en revue ces différentes modalités. Nous n'en proposons qu'une synthèse rapide. Selon la typologie choisie (cf. Gosselin, 2010), les modalités s'inscrivent sous trois axes: la réalité, la subjectivité et les institutions. Les modalités aléthiques (p. 314-324) sont celles qui renvoient à la réalité (vérité objective indépendante du jugement du locuteur : Il neige $e^{5}$ ). Au niveau des modalités ayant trait à la subjectivité on retrouve les modalités épistémiques (vérité subjective dépendante du jugement de valeur du locuteur : Cette pièce est petite) (p. 325-332), les modalités appréciatives (jugements subjectifs liés aux désirs ou aversions : Les poires, c'est bon) (p. 332-343), et les modalités bouliques (expression de désirs, volontés, souhaits, actes illocutoires directifs subjectifs : Je veux aller à la pêche ; je veux que tu sortes immédiatement!) (p. 351360). Au niveau institutionnel, nous retrouvons les modalités axiologiques (jugements de nature morale, idéologique, légale, fondés sur des systèmes et la subjectivité : Pierre est un salaud) (p. 343-351) et les modalités déontiques (jugements prescriptifs ou normes fondés sur des institutions et exprimant l'obligatoire, l'interdit, le permis, le facultatif, de nature injonctive, avec un impact sur le comportement du sujet : Essuyer ses pieds avant d'entrer) (p. 360-369).

Au vu de ces différents éléments, les textes injonctifs de notre corpus s'inscrivent sans équivoque sous la modalité déontique. Ils sont « essentiellement prescriptifs » et «à la différence des modalités bouliques, s'appuient sur des institutions » (Gosselin, 2010 : 369). En effet, dans le cas d'une recette de cuisine, d'un manuel d'entretien des équipements de laboratoire ou d'un référentiel de compétences, nous pouvons considérer que la source de l'énoncé est bien une institution. Cette distinction entre l'idéologique et l'institutionnel ne se retrouve pas chez tous les auteurs. Ainsi, pour Han (cf. 1999 : 479-483) la modalité déontique comprend à la fois les normes et les jugements fondés sur l'idéologie, ainsi que l'optatif. Les injonctions de notre corpus expriment essentiellement des consignes.

\subsection{Aspects pragmatiques}

Au niveau pragmatique, on retiendra essentiellement les notions d'acte de langage et de force illocutoire, à distinguer de la notion de modalité, comme l'explique Gosselin (cf. $2010: 18-21$ ). Ainsi que le mentionne Bracops (2005 : 14), «le langage est action en ce sens qu'il permet d'instaurer un sens, mais aussi d'agir sur le monde et sur autrui ». L'injonction, en tant qu'acte de langage, est une modalité par laquelle le locuteur demande la réalisation d'une action au destinataire. Dans le cas de notre corpus, il s'agira d'un lecteur à qui le locuteur donne des consignes à respecter, pour réaliser une recette, réaliser l'entretien et la maintenance des appareils de laboratoire, ou encore, en vue d'acquérir les compétences nécessaires à la fonction de secouriste. Cet acte possède, en outre, une force illocutoire directive.

Le contexte situationnel n'est pas non plus négligeable puisque le lecteur n'entreprend pas la lecture de ces textes comme il lirait un roman, par exemple. Dans tous les cas, il est conscient qu'il s'expose à des consignes qu'il devra suivre s'il veut atteindre le but visé : le plat présenté par la recette, l'entretien et la maintenance des appareils de laboratoire, ou encore développer les compétences requises pour devenir un secouriste. Comme le souligne Gosselin (2010 : 369) « un "bon sujet" pour une institution doit vouloir accomplir l'obligatoire et refuser l'interdit ».

\subsection{Le texte injonctif en analyse du discours}

Les spécificités du texte injonctif ont été présentées dans les différents travaux de Jean-Michel Adam (2001a, 2001b, 2004, 2005). Nous nous contentons donc, dans le cadre de cet article, de n'en mentionner que les principaux traits, notre préoccupation principale étant d'étudier l'organisation syntaxique des 
constituants. Il s'agit d'une «interaction langagière accomplie dans une situation d'énonciation impliquant des participants, une institution, un lieu, un temps et les contraintes d'une langue donnée [...]» (Adam, $2004: 36)$. Les textes injonctifs « ont en commun de dire de faire en prédisant un résultat, d'inciter très directement à l'action » (Adam, 2005 : 95). Comme le souligne encore Heurley (2001 : 30), il s'agit de textes « dont la fonction principale est de communiquer des procédures en vue d'une exécution ponctuelle [...] ou d'un apprentissage à long terme sensé permettre l'acquisition d'un savoir-faire nouveau dans un domaine particulier $»$.

Au niveau des caractéristiques, Adam (2001a : 23) relève la présence d'un lexique de spécialité propre au domaine concerné puisque la précision est de rigueur et que « la connaissance de l'univers de référence [...] est supposée commune aux co-énonciateurs ». Il note aussi (p. 24) «l'abondance de prédicats représentant des actions temporellement successives ». Les indications sur la manière de procéder ainsi que sur la durée sont aussi très présentes.

Du point de vue énonciatif, on remarque l'effacement du locuteur, ces textes émanant de figures faisant autorité, i.e. des experts. Khodabocus (2012 : 116) le mentionne à propos des recettes de cuisine :

[...] la personne de l'auteur de l'injonction n'est pas importante dans la mesure où seul le savoir culinaire compte. Le texte se voit ainsi conféré une certaine autorité.

Personne ne penserait à critiquer le contenu de la recette.

Ce fait s'applique aussi à notre corpus qui comprend, outre des recettes, un manuel et un référentiel de compétences. Ajoutons encore Adam (2001a : 23) :

Entre l'expert et le lecteur, un contrat de vérité relatif aux informations fournies est passé. Ce contrat implicite garantit au destinataire que, s'il se conforme à toutes les recommandations et s'il respecte les procédures indiquées, il atteindra le but visé.

\subsection{Le sujet de l'injonction}

Collin (2006) souligne la difficulté d'aborder la question du sujet concernant l'injonction. Dans notre cas, le problème est pour ainsi dire «double » puisque notre sujet d'étude s'intéresse à l'infinitif injonctif, forme qui devrait être dépourvue de marque de personne, et qui est à peine reconnue comme forme verbale.

En effet, le sujet est souvent caractérisé au plan morphosyntaxique. C'est lui qui donne ses marques de personne au verbe (et de genre, le cas échéant) et il se place normalement en début de phrase sous forme d'un SN. Or, dans une injonction à l'impératif, et à l'infinitif, en ce qui nous concerne, le traitement du sujet serait problématique «dans la mesure où le verbe d'une phrase impérative n'a aucun élément syntaxique qui puisse lui fournir ses traits distinctifs »(Collin, 2006 :29). Si au niveau syntaxique, impératif et infinitif partagent la particularité de ne pas faire apparaître de sujet, ces deux formes diffèrent sur le plan morphologique. À l'impératif, le sujet est repérable dans les désinences verbales alors que l'infinitif ne présente pas cette possibilité.

Pour traiter du sujet dans l'injonction, et plus particulièrement du sujet de l'infinitif injonctif, il faut donc rappeler que l'existence du sujet ne se limite pas à un sujet apparent au niveau syntaxique, mais qu'il peut s'agir d'un sujet «vide» ou [sujet Ø]. Ainsi, «l'absence matérielle de sujet se compense par la conscience d'une présence subjectale implicite»(Benayoun, 2003 : 173). L'auteur ajoute (p. 174) que « dans les cas où le sujet n'est ni visible ni audible, il y a sujet quand même ». Ce sujet coïncide avec le thème et il peut être récupéré. Avec l'infinitif injonctif, l'absence de sujet ne rend d'ailleurs pas la phrase agrammaticale. De plus, cette absence ne nuisant pas à l'interprétation du sens de l'énoncé ni à la récupération du sujet, nous pouvons considérer qu'il s'agit d'un sujet implicite et le contexte de l'énonciation permet d'en déduire qu'il s'agit d'une deuxième personne faisant référence au lecteur de l'injonction. Cette présence implicite s'inscrit d'ailleurs dans le contexte énonciatif même puisque nous 
avons affaire à une situation de communication, différée certes dans le cas du texte écrit, mais qui émane d'un locuteur à destination d'un destinataire dans le but de guider ses actions.

\subsection{Caractéristiques de l'infinitif injonctif}

L'infinitif employé dans les injonctions est un infinitif libre mot-tête d'une proposition indépendante. Dans Khodabocus (2014) nous expliquons pourquoi il faut considérer l'infinitif comme une forme verbale et non comme une forme nominale ou une catégorie double qui serait à la fois verbe et nom ${ }^{6}$. En effet, le problème réside dans le traitement de l'infinitif consistant à le présenter comme ayant des fonctions nominales alors que c'est le syntagme dont il est le mot-tête qui occupe cette fonction (cf. Vikner, 1980 ; Riegel, 2005 ; Khodabocus, 2014). Il s'agit donc d'un syntagme infinitif (SInf) (ex. 10) qui occupe une position syntaxique de SN (ex. 11) et l'infinitif conserve ses propriétés verbales, notamment la possibilité de recevoir des compléments. L'exemple (12) indique clairement que l'infinitif ne se substitue pas au nom :

(10) $[\text { Courir }]_{\text {SInf }}$ est bon pour la santé.

(11) $[\text { La course }]_{\mathrm{SN}}$ est bon pour la santé.

$(12) *[\text { Course }]_{\mathrm{N}}$ est bon pour la santé.

Si l'infinitif présente bien des cas de nominalisation, deux possibilités existent. D'une part, pour les infinitifs nominalisés en emploi libre, il n'y a pas de conversion totale au statut de nom, ce qui explique que seul le déterminant défini masculin soit accepté (le voir). De plus, tous les infinitifs n'admettent pas cet emploi (cf. Vikner, 1980 ; Kerleroux, 1996 ; Riegel, 2005). D’autre part, lorsque la conversion est complète, nous avons affaire à des infinitifs lexicalisés, qui sont d'ailleurs signalés par leur entrée propre dans le dictionnaire en tant que substantif. Seuls quelques verbes acceptent cette conversion totale et ils admettent la variation en nombre mais uniquement le genre masculin.

Outre le fait que l'infinitif ne se substitue pas au nom, nous rappellerons ici quelques caractéristiques de l'infinitif qui orientent vers une interprétation en tant que forme verbale. L'infinitif possède les propriétés verbales au niveau morphologique avec un radical et une terminaison (march-er). Au niveau syntaxique, il connaît tous les emplois distributionnels d'une forme finie. Il peut être le mot-tête d'une proposition avec sujet et/ou objet (Paul regarde les enfants jouer), il peut recevoir des adverbes (Marie espère arriver rapidement) et des compléments circonstanciels (Jean demande aux enfants de jouer dans le jardin) et il admet la négation (Ne pas laisser à la portée des enfants). Le sujet de l'infinitif est, quant à lui, restituable au niveau syntaxique. Il ne s'agit donc que d'une absence morphologique de la marque de sujet et non d'une absence totale du sujet qui justifierait qu'on parle de forme impersonnelle.

Dans les injonctions, l'infinitif exprime un ordre, un conseil, une consigne, entre autres. On le retrouve dans différents textes comme les modes d'emploi de divers produits, les recettes de cuisine, le code de la route, les libellés de conseil, les manuels, les référentiels de compétences, etc. Ce type de texte s'inscrivant dans une situation de communication, et s'adressant forcément à un destinataire, nous pouvons en déduire que l'infinitif n'est pas une forme « impersonnelle ». De plus, l'emploi de l'infinitif injonctif n'existant qu'à l'écrit en français contemporain, nous avons une indication que le sujet implicite est obligatoirement humain, à moins de supposer que les animaux soient dotés de la faculté de lecture. Les ordres exprimés à l'infinitif ne s'adressent pas à un sujet particulier, mais à tous les lecteurs susceptibles de réaliser le procès :

«[...] il y a parcours sur tous les sujets susceptibles d'instancier la place du terme de départ de la relation. Par conséquent, tout «lecteur», qui est donc un sujet potentiel du procès, s'identifie à ce sujet et nous arrivons à l'interprétation de l'énoncé en tant qu'injonction "de portée générale », ce qui convient parfaitement aux instructions dans les modes d'emploi [...]. » (Turner, $2000: 13$ ) 


\section{3 Étude de cas}

Dans cette partie, nous faisons une étude de cas sur corpus afin d'étudier l'organisation syntaxique des constituants autour de l'infinitif dans trois types de textes : les recettes de cuisine, un manuel d'entretien et de maintenance des équipements de laboratoire et un référentiel de compétences du secouriste. Nous expliquons dans un premier temps la méthodologie adoptée. Dans un second temps, nous analysons les données en considérant l'occurrence des différents verbes et la structuration syntaxique. Nous comparons les résultats pour les trois catégories. Enfin, nous proposons une interprétation de ces données en tenant compte de l'aspect théorique de l'injonction développé dans la partie précédente.

\subsection{Méthodologie}

La base de données utilisée ayant été construite par nos soins, la méthodologie est assez particulière. Nous expliquons le choix du corpus et comment nous avons procédé pour la constitution de ce dernier.

\subsubsection{Choix du corpus}

Nous avons recueilli les données à partir d'un livre de cuisine, un livre de desserts, un manuel d'entretien et de maintenance et un référentiel de compétences dans le but, d'une part, de relever les structurations syntaxiques des constituants du texte injonctif, et d'autre part, de voir si des différences existent selon la « catégorie » du texte injonctif.

Pour l'organisation syntaxique des constituants des phrases, nous nous sommes basée sur un dictionnaire des verbes, le Dictionnaire des verbes du français actuel. Constructions, emplois, synonymes (FLOREA, L. S., FUCHS, C., \& MÉLANIE-BECQUET, F., 2010). Cet ouvrage a l'intérêt de ne recenser que les verbes les plus courants de la langue contemporaine et le classement par structure se fait selon le sens en contexte. Nous avons ainsi une seule entrée par verbe, avec les différentes organisations syntaxiques pour chaque sens du verbe en question.

\subsubsection{Construction de la base de données}

Pour la construction de la base de données, nous avons recueilli les phrases que nous avons entrées dans un tableau Excel, selon le modèle du Tableau 1 ci-dessous :

\begin{tabular}{|c|c|c|c|c|c|c|c|c|c|c|c|c|}
\hline $\mathrm{m}=$ Catégorie & FSource & Fxemples & Verbe & Constit & truc_dic $\mathbf{F}$ & Struc & & & CC1 & . & EC & $\begin{array}{l}\text { CC1_D } \\
\text { épl }\end{array}$ \\
\hline 1 Recette & $\begin{array}{l}\text { nos } 200 \text { meilleurs } \\
\text { desserts et biscuits }\end{array}$ & $\begin{array}{l}\text { au robot culinaire ou au mélangeur, moudre } \\
36 \text { finement la moitié des amandes }\end{array}$ & moudre & $\begin{array}{l}(S P)-V- \\
(A d v)-S N\end{array}$ & $V+S N$ & $\mathrm{~V}+\mathrm{SN}$ & $S P$ & là & antép & moyen & oui & oui \\
\hline 2 Recette & $\begin{array}{l}\text { nos } 200 \text { meilleurs } \\
\text { desserts et biscuits }\end{array}$ & 36 réserver & réserver & V & $V+S N$ & IV & & & & & & \\
\hline 3 Recette & $\begin{array}{l}\text { nos } 200 \text { meilleurs } \\
\text { desserts et biscuits }\end{array}$ & $\begin{array}{l}\text { dans un grand bol, à l'aide d'un batteur électrique, } \\
\text { battre le beurre, le sucre et la cassonade jusqu'à ce } \\
36 \text { que le mélange soit léger }\end{array}$ & battre & $\begin{array}{l}\text { (SP) - (SP)- } \\
\text { V-SN- } \\
\text { (PCIRC) }\end{array}$ & $V+S N$ & $\mathrm{~V}+\mathrm{SN}$ & SP & dans & antép & lieu & ? & oui \\
\hline 4 Recette & $\begin{array}{l}\text { nos } 200 \text { meilleurs } \\
\text { desserts et biscuits }\end{array}$ & $\begin{array}{l}\text { ajouter les œufs un à un, en battant bien entre } \\
36 \text { chaque addition }\end{array}$ & ajouter & $\begin{array}{l}\text { V-SN- } \\
(\text { Adv) - } \\
\text { (PCIRC) }\end{array}$ & $\mathrm{V}+\mathrm{SN}$ à SN & $\mathrm{V}+\mathrm{SN}$ & Adv & & postP & manière & ? & non \\
\hline 5 Recette & $\begin{array}{l}\text { nos } 200 \text { meilleurs } \\
\text { desserts et biscuits }\end{array}$ & 36 incorporer la vanille et le lait & incorporer & $V-S N$ & $\mathrm{~V}+\mathrm{SN}$ à SN & $\mathrm{V}+\mathrm{SN}$ & & & & & & \\
\hline
\end{tabular}

Tableau 1 : Base de données

Nous avons d'abord transcrit les phrases du corpus telles qu'elles apparaissent (colonne «Exemples »). Ensuite, dans les cas de juxtaposition ou de coordination, l'entrée se retrouve sur deux lignes ou plus (selon le nombre de propositions présentes), pour permettre d'analyser chaque verbe. Par exemple, 
(13) Ajouter la chair de crabe et faire sauter pendant 1 minute. (Recette)

sera analysé en deux propositions, sur deux lignes de la base de données (Ajouter la chair de crabe / faire sauter pendant 1 minute).

Pour l'annotation, nous avons spécifié la catégorie (recette, manuel, référentiel), la source et le numéro de page. Nous avons ensuite relevé le verbe.

Concernant la structuration proprement dite, nous avons trois colonnes différentes. Dans un premier temps, nous avons relevé les constituants immédiats de la phrase (colonne «Constit» du Tableau 1). Nous faisons figurer entre parenthèses les constituants qui ont la fonction de circonstant. Pour cette étape, nous ne distinguons pas les circonstants arguments et les non-arguments. Nous relevons simplement les constituants qui indiquent, d'un point de vue sémantique une « circonstance » (cf. G. Gross : 192 ; Riegel et al., 2009). Ainsi, pour l'exemple (14), nous avons dans la colonne «Constit » la structure [V - (SP) $\mathrm{SN}-(\mathrm{SP})-(\mathrm{SP})]$ :

$(14)[\text { Dégager }]_{\mathrm{V}}[\text { en urgence }]_{(\mathrm{SP})}[\text { une victime }]_{\mathrm{SN}}[\text { de la zone de danger }]_{(\mathrm{SP})}$ [en toute sécurité $]_{(\mathrm{SP})}$ (Référentiel de compétences)

Ensuite, nous avons recueilli les structures proposées dans le Dictionnaire des verbes pour le sens du verbe qui nous intéressait (colonne «Struc_dic»). Pour le verbe « dégager », le sens pour l'exemple (14) est «sortir ; extraire » et le dictionnaire donne comme illustration sortir un cadavre des ruines, et une structure [V + SN de SN] (cf. Florea, Fuchs, Mélanie-Becquet, 2010 : 67). C'est cette structure que nous faisons figurer dans la colonne «Struc_dic ». Il s'agit de la structure argumentale canonique pour le sens précis du verbe en question où figurent tous les arguments.

Enfin, nous avons synthétisé les données de ces deux colonnes afin de recenser les structures effectives observées dans le corpus (colonne «Struc»). Dans cette entrée, seuls les arguments obligatoires sont représentés. Les SP objets indirects du verbe sont notés «SPO » et les SP circonstants obligatoires sont notés «SPC» pour permettre leur analyse. Pour les phrases présentant plusieurs circonstants dans la colonne «Constit», les données de la colonne «Struc_dic»permettent de déterminer lequel est aussi argument. La colonne «Struc » indiquera $[\mathrm{V}+\mathrm{SN}+\mathrm{SPC}]$ pour l'exemple (14), où SPC renvoie au SP [de la zone de danger], qui correspond au constituant [de $\mathrm{SN}]$ dans «Struc_dic ».

Nous avons ensuite une série de colonnes pour le traitement des compléments circonstanciels où nous avons codé la catégorie $(A d v, S P, S N \ldots)$, la position par rapport au verbe (antéposé ou postposé), la circonstance exprimée (moyen, lieu, temps, manière...) et le comportement face aux tests d'effacement et de déplacement. L'annotation se fait selon le principe d'un paramètre par colonne.

\subsection{Exploitation des données}

\subsubsection{Occurrences des verbes}

Notre corpus compte 92 occurrences (30 de la catégorie « recette », 31 de la catégorie « manuel » et 31 de la catégorie « référentiel »). Ces propositions s'organisent autour de 57 verbes différents, toutes catégories confondues (15 verbes apparaissent dans les recettes, 18 dans le manuel et 25 dans le référentiel de compétences, et certains verbes apparaissent dans plus d'une catégorie). Pour chaque verbe relevé, nous avons spécifié la catégorie (recette, manuel, référentiel) et nous avons vérifié leur appartenance ou non au domaine de spécialité concerné selon les définitions du TLFi (art culinaire, médecine, techniquelélectricité), ces précisions étant données dans ce dictionnaire. De manière générale, moins de $20 \%$ des 57 verbes de notre corpus appartiennent au lexique de spécialité, les trois catégories de textes confondues. 
En considérant les occurrences par catégorie (55 verbes qui apparaissent dans une seule des catégories concernées), les verbes se répartissent comme suit : 43,6\% des 55 verbes apparaissent dans le référentiel, $30,9 \%$ dans le manuel et $25,5 \%$ dans les recettes. En utilisant le critère de l'appartenance ou non à un lexique de spécialité, nous constatons que les verbes présents dans les recettes sont ceux ayant le plus fort taux d'appartenance au champ sémantique de l'art culinaire, comparé au manuel et au référentiel, pourtant des écrits plus «techniques ». La répartition des verbes, selon qu'ils appartiennent ou pas au champ sémantique du domaine, est illustrée dans le graphique suivant :

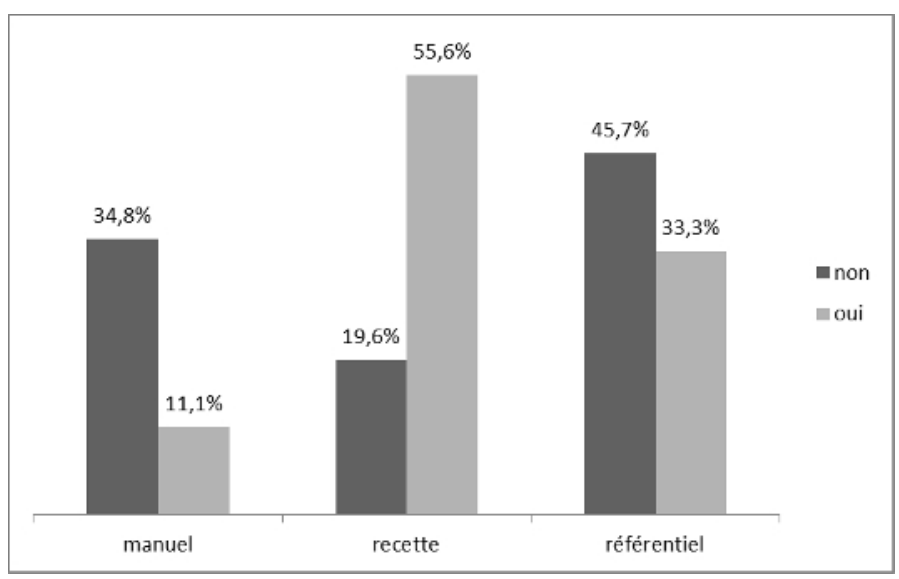

Graphique 1 : Répartition des verbes selon le champ sémantique du domaine de spécialité

Ainsi, plus de 55\% des verbes appartenant à un champ sémantique précis sont utilisés dans les recettes, alors que pour le manuel d'entretien et de maintenance et le référentiel de compétences, nous trouvons majoritairement des verbes n'appartenant pas à un lexique de spécialité. Cependant, dans ces deux dernières catégories, le lexique de spécialité est présent sous forme nominale (détresse vitale, capteur optique...).

\subsubsection{Organisation des constituants}

Pour analyser l'organisation des constituants, nous considérons les trois colonnes « Constit», «Struc_dic » et «Struc » séparément et nous comparons aussi pour chacune d'elles les répartitions par catégorie.

\section{- Colonne « Constit»}

Ici, nous notons l'organisation des constituants tels qu'ils apparaissent dans la proposition concernée. Ainsi, Régler le sélecteur de température sur la température ambiante (OMS, 2008 : 16) sera noté [V $\mathrm{SN}-(\mathrm{SP})]$, les parenthèses indiquant un circonstant comme expliqué au §3.1.2. Nous avons relevé 27 organisations différentes toutes catégories confondues, mais uniquement deux organisations avec une occurrence supérieure ou égale à $5 \%$ du nombre total des organisations : [V - SN] avec 35,9\% et [V$\mathrm{SN}-(\mathrm{SP})]$ avec $23,9 \%$.

En considérant l'organisation syntaxique par catégorie, nous obtenons la répartition suivante :

- 10 organisations dans le manuel d'entretien et de maintenance ;

- 13 dans les recettes ;

- 8 dans le référentiel de compétences. 
Nous avons relevé les organisations avec une occurrence supérieure ou égale à $5 \%$ du total et en les triant par catégorie, nous obtenons la répartition suivante :

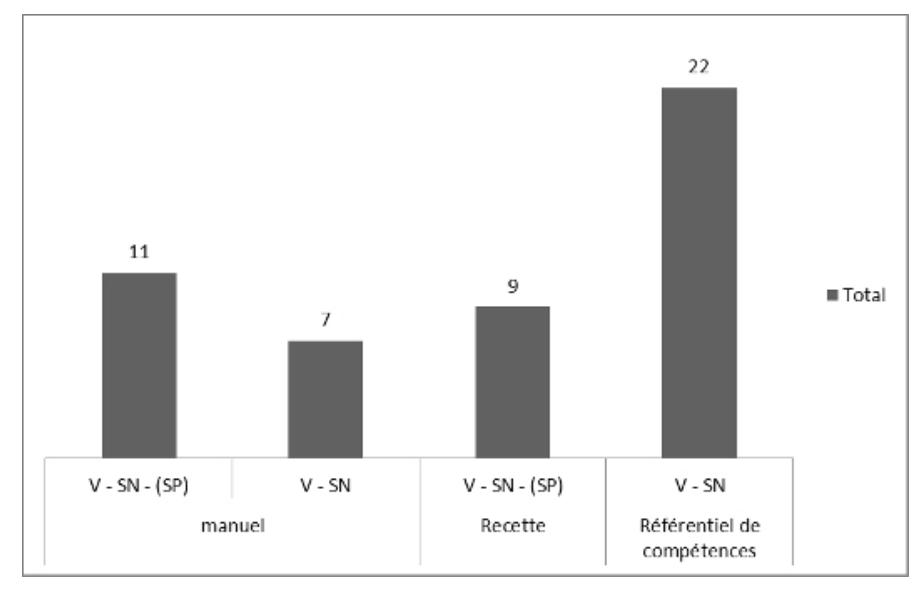

Graphique 2 : Organisation des constituants par catégorie

Dans le manuel (ex. 15) et les recettes (ex. 16), l'organisation la plus utilisée est [V - SN - (SP)] $(35,5 \%$ du total des propositions utilisées dans le manuel et $30 \%$ des propositions utilisées dans les recettes), alors que dans le référentiel de compétences (ex. 17) on trouve essentiellement [V - SN] (71\% des propositions du référentiel présentent cette organisation) :

(15) Lire le pH de la solution sur l'échelle de lecture (cadran) ou l'écran du pH-mètre. (Manuel)

(16) Déposer les biscuits sur des grilles [...]. (Recette)

(17) Alerter les secours appropriés. (Référentiel)

\section{- Colonne «Struc_dic »}

Cette colonne nous donne la distribution canonique des constituants autour du verbe, pour le sens concerné. Elle contient 13 organisations différentes, toutes catégories confondues, et trois organisations avec une occurrence supérieure ou égale à $5 \%$ du total des organisations : [V + SN] 59,3\%, $[\mathrm{V}+\mathrm{SN}$ prép $\mathrm{SN}] 9,9 \%$ et $[\mathrm{V}+\mathrm{SN}$ à $\mathrm{SN}] 6,6 \%{ }^{7}$

En considérant les différentes catégories, nous obtenons la répartition suivante :

- 8 organisations dans le manuel ;

- 4 dans les recettes ;

- 5 dans le référentiel.

Les occurrences de l'ordre de $5 \%$ ou plus du nombre total des organisations syntaxiques sont indiquées dans le graphique suivant pour chaque catégorie : 


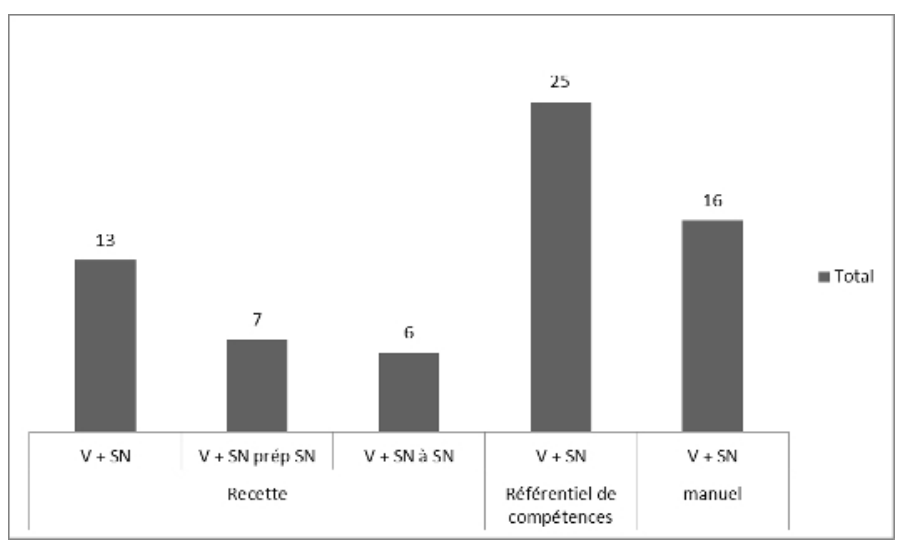

Graphique 3 : Structure canonique des verbes par catégorie

Dans les trois catégories, on trouve majoritairement des verbes dont la structure canonique est [V $+\mathrm{SN}]$, qui représente un verbe transitif avec objet direct (réserver, indiquer, surveiller...).

Cependant, comme nous pouvons le constater dans le Graphique 3, dans le dictionnaire on trouve [V + SN prép SN] mais aussi des occurrences pour lesquelles la préposition est précisée : [V + $\mathrm{SN}$ à $\mathrm{SN}]$, $[\mathrm{V}+\mathrm{SN}$ sur $\mathrm{SN}]$, etc ${ }^{8}$. En remplaçant toutes les prépositions par [prép], nous ramenons le nombre d'organisations à 7 , toutes catégories confondues, les plus récurrentes étant $[\mathrm{V}+\mathrm{SN}]$ avec $59,3 \%$ et [V + SN prép SN] avec $27,5 \%$ du total respectivement. La répartition par catégorie est alors : 4 organisations chacunes pour le manuel et le référentiel de compétences et 3 pour les recettes. Les occurrences qui comptent $5 \%$ ou plus du total des organisations sont indiquées dans le Graphique 4 pour chaque catégorie :

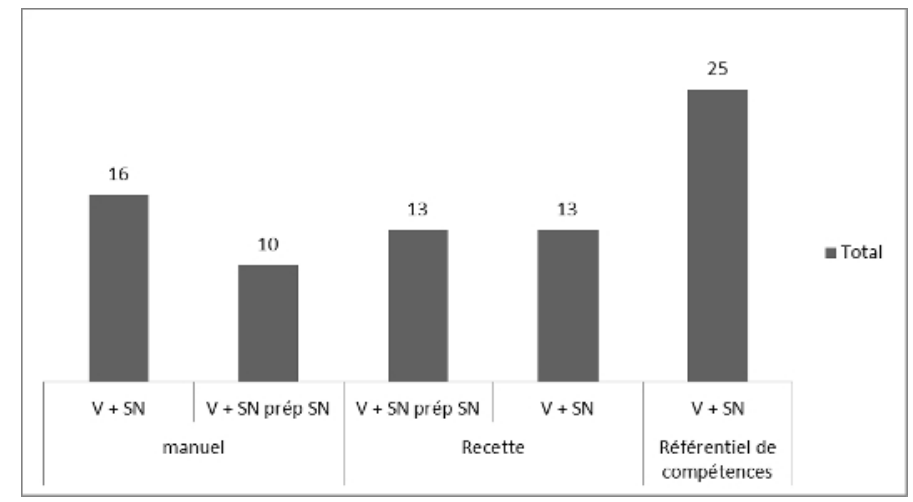

Graphique 4 : Structure canonique simplifiée des verbes par catégorie

Si la structure syntaxique la plus récurrente demeure $[\mathrm{V}+\mathrm{SN}]$ pour les trois catégories de textes $(80,6 \%$ des propositions du référentiel, 53,3\% de celles du manuel et $43,3 \%$ de celles des recettes), nous constatons que dans les recettes, cette structure est aussi fréquente que la structure [V + SN prép SN] et que cette organisation est aussi très utilisée dans le manuel (33,3\% des propositions du manuel). Ces données, nous le rappelons, n'indiquent cependant que l'organisation canonique des verbes, et les données de la colonne suivante «Struc » nous permettra de vérifier si cela correspond aux organisations effectivement utilisées. 


\section{- Colonne « Struc»}

Cette dernière colonne contenant l'organisation syntaxique des constituants recense la structure effective relevée, en se basant sur la structure canonique, et en ne faisant figurer que les constituants se trouvant dans de la structure argumentale du verbe (verbe V, objet direct SN, objet indirect SPO, circonstant argument SPC). Nous avons compté 9 structures différentes, toutes catégories confondues, les plus fréquentes $(\geq 5 \%$ du nombre total) étant $[\mathrm{V}+\mathrm{SN}]$, avec $60,9 \%,[\mathrm{~V}+\mathrm{SN}+\mathrm{SPC}]$, avec $18,5 \%$ et $[\mathrm{V}]$, avec $10,9 \%$.

En considérant les différentes catégories, nous obtenons la répartition suivante :

- 4 structures dans le manuel ;

- 4 dans les recettes ;

- 6 dans le référentiel.

Les occurrences de l'ordre de $5 \%$ ou plus du total sont indiquées dans le graphique suivant pour chaque catégorie :

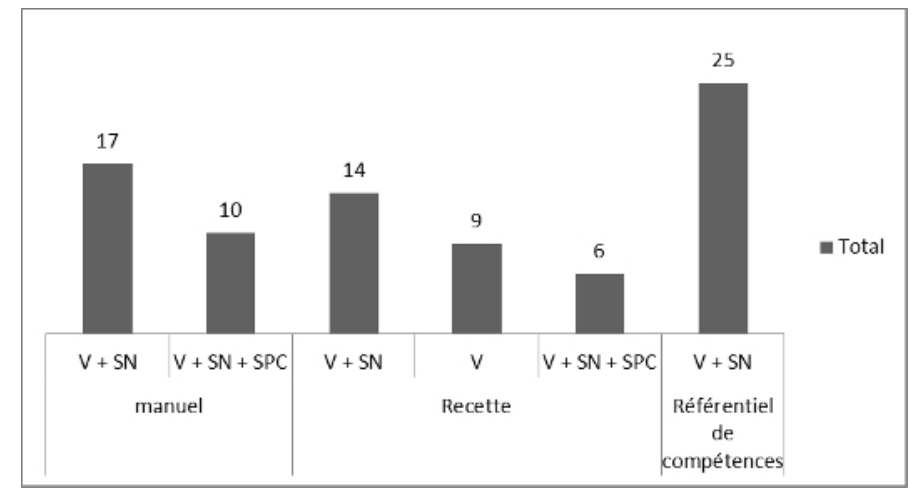

Graphique 5 : Structures relevées comprenant le verbe et les arguments obligatoires

Dans les trois catégories, la structure la plus utilisée est $[\mathrm{V}+\mathrm{SN}](80,7 \%$ des occurrences du référentiel, $54,8 \%$ de celles du manuel et $46,7 \%$ de celles des recettes). Il faut toutefois rappeler ici que cette colonne ne recensant que les arguments obligatoires, nous ne retrouvons pas les compléments circonstanciels non arguments qui peuvent accompagner les propositions concernées. Sous cette annotation, on retrouvera, outre le verbe et, éventuellement, son objet (dont le circonstant argument) des circonstants non obligatoires :

(18) Examiner la victime. (Référentiel)

(19) Prendre les mesures de protection nécessaires pour éviter la transmission des infections. (Référentiel)

(20) Dans un grand bol, à l'aide d'un batteur électrique, battre le beurre, le sucre et la cassonade jusqu'à ce que le mélange soit léger. (Recette)

Cette colonne fait aussi ressortir une particularité des recettes au niveau de l'organisation syntaxique. En effet, on note que dans les recettes, on peut trouver [V] (30\% des propositions dans les recettes de notre corpus). Le verbe est ici en emploi absolu, ce qui en soi n'est pas problématique, si ce n'est que la recette permet cet emploi pour des verbes qui n'admettent pas cette construction à la forme finie, sauf à l'impératif : 
(21) Réserver / *Jean réserve / Jean réserve les amandes / Réservez.

La recette se rapprochant plus du texte procédural, on va à l'essentiel et le complément du verbe déjà mentionné dans le cotexte antérieur n'est pas repris :

(22) $\mathrm{Au}$ robot culinaire ou au mélangeur, moudre finement la moitié des amandes. Réserver. (Recette)

On trouve cette même particularité avec les structures à double objet. En effet, dans le texte injonctif, le second complément n'est pas repris :

(23) Incorporer la vanille et le lait. (Recette)

Le verbe incorporer présente une structure $[\mathrm{V}+\mathrm{SN}$ à $\mathrm{SN}]$ (ajouter quelque chose à quelque chose). Or, dans le texte où il apparaît (une recette), il s'agit d'ajouter les éléments du [SN] la vanille et le lait à un mélange déjà mentionné dans le cotexte antérieur.

Si nous ne considérons que les occurrences d'ordre supérieur ou égal à $5 \%$ du total, on constate que toutes catégories confondues, $[\mathrm{V}+\mathrm{SN}]$ de «Struc » correspond à $[\mathrm{V}-\mathrm{SN}]$ ou $[\mathrm{V}-\mathrm{SN}-(\mathrm{SP})] \mathrm{de}$ « Constit ». En ajoutant le critère du texte en question, on note que les structures des deux colonnes se recoupent dans toutes les réalisations avec une occurrence $\geq 5 \%$, comme indiqué dans le graphique suivant ${ }^{9}$ :

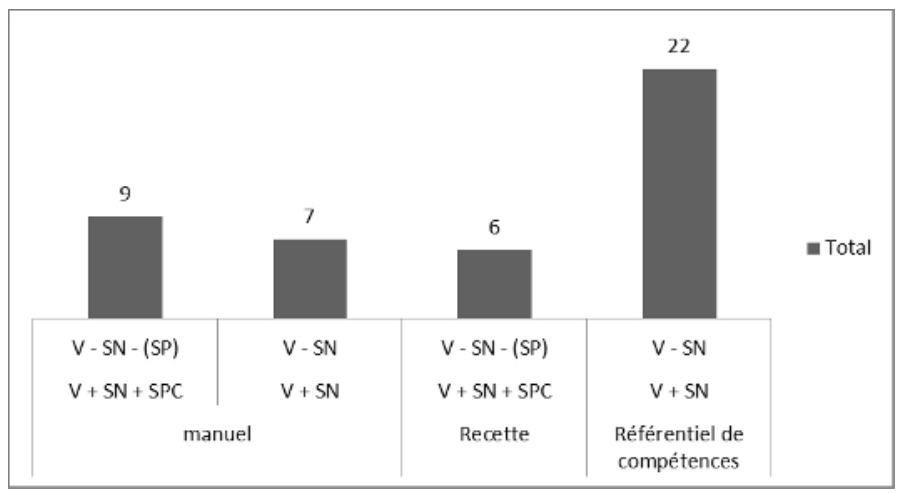

Graphique 6 : Correspondances des structures de «Struc » et de «Constit » par type de texte

\section{- Comportement des compléments circonstanciels}

Pour analyser le comportement des compléments circonstanciels (désormais $C C$ ) dans notre corpus, nous prenons en considération le nombre par proposition, la catégorie (adverbe, SN, SP...), la position par rapport au verbe (antéposé ou postposé), la circonstance exprimée (manière, but, lieu, temps...) et les tests d'effacement et de déplacement.

Le nombre de circonstants par proposition varie de 1 à 3 dans notre corpus. Pour leur identification, nous les avons indexés selon leur ordre d'apparition $\left(C C_{1}, C C_{2}, C C_{3}\right)$. Des 92 propositions analysées, 42 ne comportent aucun complément circonstanciel, 31 occurrences comptent un unique $\mathrm{CC}, 13$ occurrences comptent deux CC et 6 occurrences comptent trois CC. Nous considérerons les différents CC séparément selon leur position. 


\section{- Position des circonstants par rapport au verbe}

Lorsque la proposition contient un unique CC (31 occurrences de notre corpus), la répartition selon la position par rapport au verbe est la suivante : $93,5 \%$ en position postposée au verbe (post $P$ ) et $6,5 \%$ en position antéposée (antéP). En ajoutant la catégorie du texte comme critère, on constate que toutes les réalisations en position antéposée au verbe se trouvent dans les recettes. Toutes positions confondues, les recettes sont les textes comportant le plus de $\mathrm{CC}_{1}$ et le référentiel, celui qui en compte le moins. $48,4 \%$ des occurrences présentent un circonstant argument et ils sont tous postposés au verbe. Le graphique suivant indique la position de $\mathrm{CC}_{1}$ pour chaque catégorie :

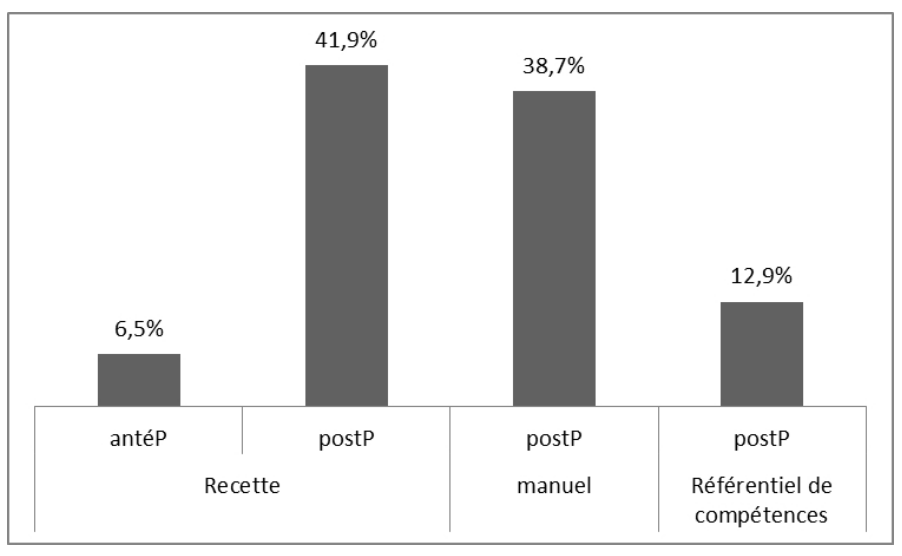

Graphique 7 : Position $\mathrm{CC}_{1}$ par catégorie

Nous avons relevé 13 propositions avec deux $\mathrm{CC}$. Tous les $\mathrm{CC}_{2}$ sont postposés au verbe et seules deux occurrences comptent un CC argument. Dans 30,8\% des cas, $\mathrm{CC}_{1}$ est antéposé au verbe (Si nécessaire, nettoyer les parties externes avec un chiffon imbibé de détergent doux). On les trouve dans le manuel d'entretien (23,1\% des occurrences) et dans les recettes $(7,7 \%)$. On constate aussi que dans le référentiel de compétences, une seule proposition compte deux CC, les deux postposés au verbe. On rencontre plus de $50 \%$ des occurrences avec deux $\mathrm{CC}$ dans le manuel d'entretien. Le graphique 8 indique la répartition de $\mathrm{CC}_{2}$ par position en considérant la position de $\mathrm{CC}_{1}$ et la catégorie du texte (par exemple, la première colonne du graphique indique que dans le manuel, on trouve $\mathrm{CC}_{1}$ en position antéP et $\mathrm{CC}_{2}$ en position post $P$ et que cette séquence représente $23,1 \%$ des occurrences avec deux $\mathrm{CC}$ ) :

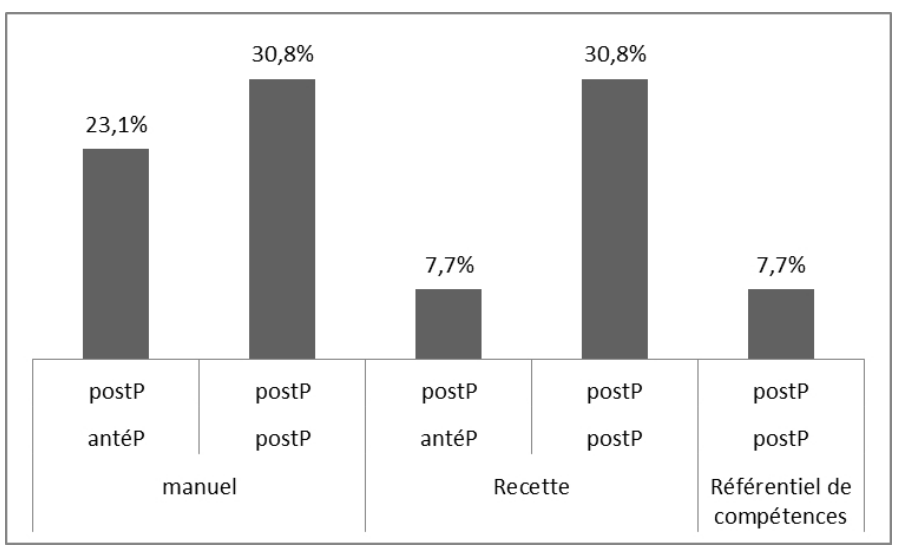

Graphique 8 : Position $\mathrm{CC}_{2}$ selon position $\mathrm{CC}_{1}$ et catégorie 
Six occurrences de notre corpus comptent trois CC. Dans tous les cas $\mathrm{CC}_{3}$ est postposé au verbe. Nous comptons deux occurrences où $\mathrm{CC}_{1}$ et $\mathrm{CC}_{2}$ sont antéposés au verbe (dans les recettes). Une seule proposition comprenant trois $\mathrm{CC}$ présente un $\mathrm{CC}$ argument. Le graphique suivant indique la répartition en considérant la position des $\mathrm{CC}$ et la catégorie du texte (chaque colonne indique, de bas en haut, la catégorie du texte et les positions de $\mathrm{CC}_{1}, \mathrm{CC}_{2}$ et $\mathrm{CC}_{3}$ respectivement) :

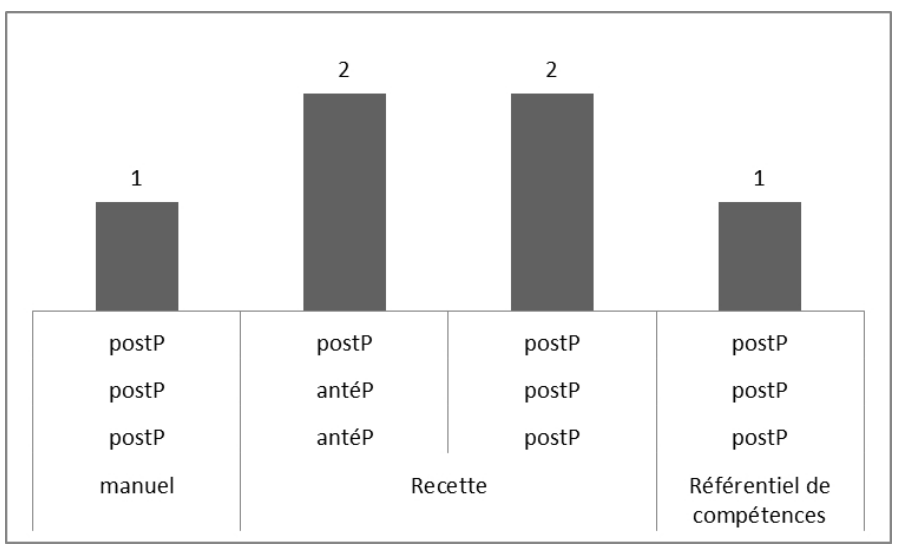

Graphique 9 : Répartition des positions des CC selon la catégorie

On peut constater que peu importe le nombre de circonstants dans la proposition, la position postP est privilégiée. On trouve très rarement des $\mathrm{CC}$ en position antéP dans les textes injonctifs de notre corpus. Nous nous intéresserons dans la partie suivante aux circonstances exprimées par ces différents CC.

\section{○ Circonstances exprimées}

Les circonstances les plus exprimées par $\mathrm{CC}_{1}$ sont le lieu $\left(48,4 \%\right.$ des occurrences de $\left.\mathrm{CC}_{1}\right)$, le temps $(19,4 \%)$, le moyen $(9,7 \%)$ et la manière $(9,7 \%)$. Nous avons aussi considéré la catégorie concernée. Dans les recettes, on trouve principalement le lieu et le temps; dans le manuel d'entretien, on trouve le lieu et le moyen. Dans le référentiel, les circonstances sont le temps, la manière, le but et la condition (une occurrence par circonstance). Le graphique suivant indique la répartition des circonstances selon la catégorie du texte :

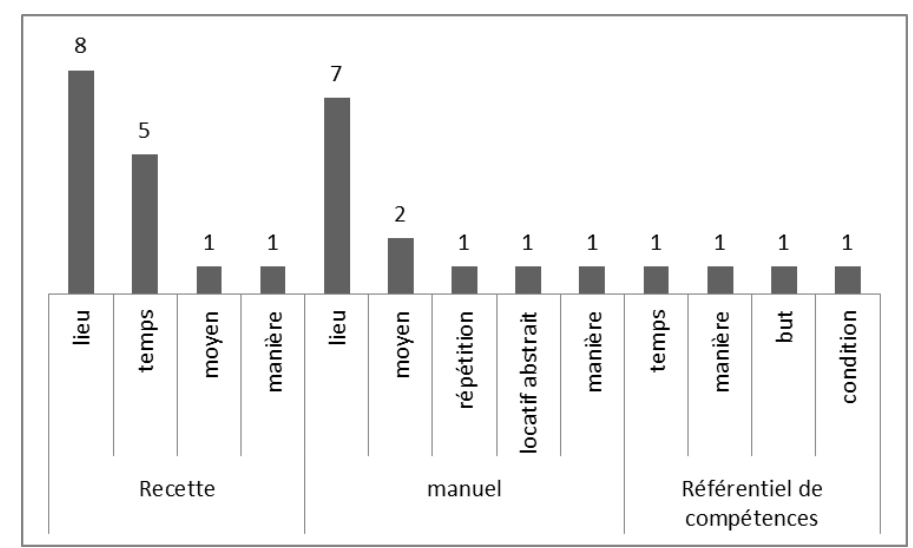

Graphique 10 : Circonstance exprimée par $\mathrm{CC}_{1}$ selon la position et la catégorie 
Dans les recettes et le manuel, le lieu est la circonstance la plus exprimée (déposer les plaques [sur des grilles], mettre le sélecteur [sur la position $p H]$ ]...). Les indications de temps sont aussi très présentes dans les recettes, les durées de préparation, de cuisson, etc. étant nécessaires à la réussite du plat (à la micuisson, pendant 1 minute...). Lorsque $\mathrm{CC}_{1}$ est en position antéP, on le trouve dans les recettes (deux occurrences) et il exprime le moyen et le lieu.

Les expressions les plus exprimées par $\mathrm{CC}_{2}$ sont la manière (finement, en faisant attention...) dans 38,5\% des propositions avec deux CC et le but (jusqu'à ce que la pâte soit homogène...) dans 23,1\% des occurrences. Nous avons aussi relevé les circonstances exprimées par $\mathrm{CC}_{1}$ et $\mathrm{CC}_{2}$ dans les propositions avec deux CC.

Les séquences $\left[\mathrm{CC}_{1}-\mathrm{CC}_{2}\right.$ les plus fréquentes sont [moyen - manière] (Au robot culinaire ou au mélangeur, moudre finement la moitié des amandes) et [condition - moyen] (Si nécessaire, nettoyer les parties externes avec un chiffon imbibé de détergent doux). Le graphique suivant indique les séquences $\left[\mathrm{CC}_{1}-\mathrm{CC}_{2}\right]$ ainsi que les textes où ils apparaissent (de bas en haut, la catégorie du texte, la circonstance exprimée par $\mathrm{CC}_{1}$ et celle exprimée par $\mathrm{CC}_{2}$ ) :

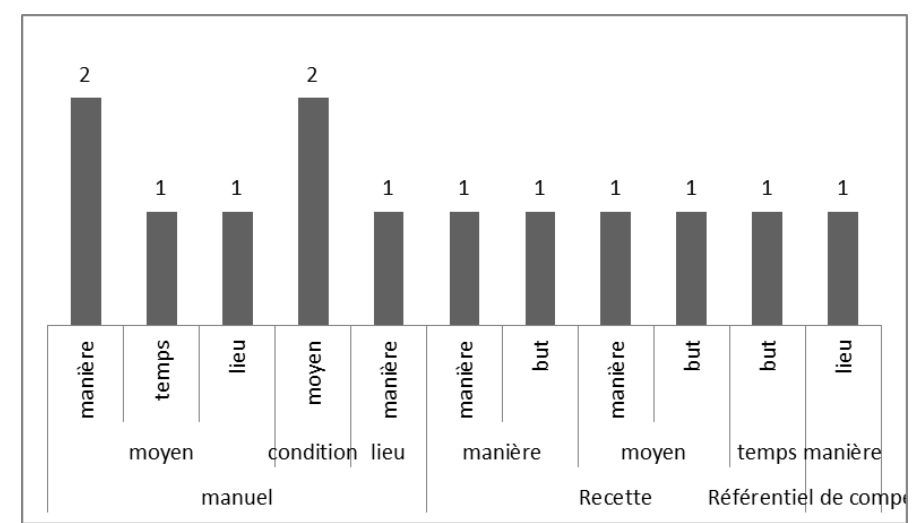

Graphique 11 : Circonstances exprimées par $\left[\mathrm{CC}_{1}-\mathrm{CC}_{2}\right]$ selon la catégorie du texte

Lorsque la proposition compte trois $\mathrm{CC}$, les circonstances exprimées par $\mathrm{CC}_{3}$ sont le but (4 occurrences) et le moyen ( 2 occurrences).

\section{- Tests de déplacement et d'effacement}

Le test de déplacement de $\mathrm{CC}_{1}$ indique que dans $67,7 \%$ des cas où la proposition compte un $\mathrm{CC}$, ce circonstant ne se déplace pas dans la proposition (Rincer l'électrode à l'eau distillée / ?? À l'eau distillée, rincer l'électrode / ??Rincer à l'eau distillée l'électrode). Lorsque le déplacement est possible, nous avons constaté que des restrictions pouvaient s'appliquer. Ainsi, dans :

(24) Prendre les mesures de précaution nécessaires [pour éviter la transmission des infections],

le CC peut se déplacer, mais en position antéposée par rapport au verbe (??Prendre, pour éviter la transmission des infections, les mesures [...]).

Lorsque la proposition comprend deux $\mathrm{CC}$, nous constatons que $\mathrm{CC}_{2}$ ne peut pas se déplacer dans $53,9 \%$ des cas et dans $30,8 \%$ des cas, il ne peut se déplacer qu'en position post $V$. Nous avons aussi comparé les comportements de $\mathrm{CC}_{1}$ et $\mathrm{CC}_{2}$ lorsque la proposition compte deux $\mathrm{CC}$. La répartition est indiquée dans le graphique suivant (de bas en haut : comportement de $\mathrm{CC}_{1}$ suivi de celui de $\mathrm{CC}_{2}$ ) : 


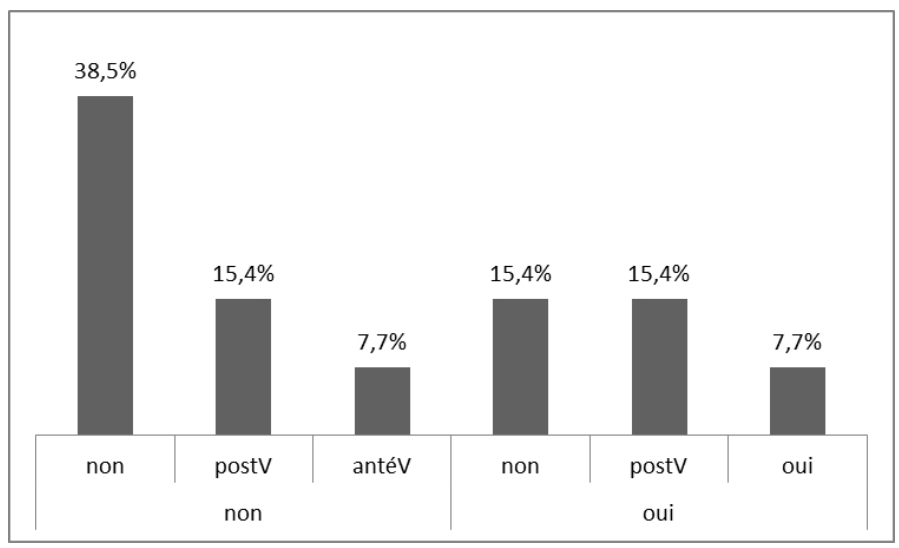

Graphique $12: \mathrm{CC}_{1}$ et $\mathrm{CC}_{2}$ face au test de déplacement

Dans $61,5 \%$ des cas, $\mathrm{CC}_{1}$ ne se déplace pas. Dans ce cas, le comportement de $\mathrm{CC}_{2}$ est très contraint : $38,5 \%$ des occurrences de $\mathrm{CC}_{2}$ ne se déplacent pas, et pour les cas où ce déplacement est possible, la position par rapport au verbe est contrainte. On constate aussi que lorsque $\mathrm{CC}_{1}$ est mobile, $\mathrm{CC}_{2}$ préfère ne pas se déplacer, ou alors principalement en position postverbale (postV). Dans tous les cas, peu importe la catégorie du texte, on constate que $\mathrm{CC}_{2}$ n'est pas très mobile dans la phrase et qu'il se place très difficilement en position anté $V$.

Une seule occurrence de $\mathrm{CC}_{3}$ est déplaçable dans la proposition. Pour l'occurrence où le déplacement est possible, $\mathrm{CC}_{3}$ ne se déplace qu'en position antéV (Nettoyer le laveur à l'eau distillée après emploi, [pour éliminer les restes de sels dans les canaux des sous-systèmes de distribution et d'extraction] / [Pour éliminer les restes de sels [...], nettoyer le laveur [...] / ??Nettoyer, [pour éliminer les restes de sels [...], le laveur $[\ldots])$.

Pour le test d'effacement, lorsque la proposition compte un seul $\mathrm{CC}$, on relève une seule occurrence où c'est possible sans changement important du sens de la proposition (Laisser refroidir (complètement)). Pour les $\mathrm{CC}_{1}$ arguments (48,4\% des occurrences comptant un CC), l'effacement est impossible dans le contexte de l'injonction. Concernant les 48,4\% restants, si l'effacement ne donne pas une proposition agrammaticale, l'information apportée par le CC ne semble pas être facultative, mais nécessaire au bon déroulement du procès (ainsi, pour faire sauter pendant 1 minute, le résultat ne sera pas le même sans le $\mathrm{CC})$.

$\mathrm{CC}_{2}$ est supprimable pour une seule occurrence présentant deux CC. Dans 84,6\% des cas, si la suppression n'amène pas une proposition agrammaticale, le cadre de l'injonction bloque l'effacement puisque l'information apportée par le circonstant en question est une donnée importante (S'ils sont sales, nettoyer la fenêtre des émetteurs de lumière et des capteurs [avec une petite brosse]).

Dans les six propositions avec trois $\mathrm{CC}$, l'effacement de $\mathrm{CC}_{3}$ est possible grammaticalement mais impossible contextuellement.

\subsection{Interprétation des données}

Les données recueillies et analysées indiquent premièrement que tous les verbes de notre corpus sont des prédicats actionnels, conformément aux résultats des travaux sur l'analyse du discours. 
Dans ce travail, nous nous sommes principalement intéressée à l'organisation syntaxique des constituants autour d'un infinitif injonctif. Or, il ressort de nos différentes analyses que l'infinitif se comporte comme toute forme verbale, et peut recevoir des compléments d'objet, des compléments circonstanciels, et admet la négation. Le sujet est récupérable et le contexte de l'injonction, dont nous avons présenté les caractéristiques principales, permet d'interpréter ce sujet comme possédant le trait [+humain]. Ce trait est confirmé pour tous les verbes de notre corpus.

En relevant les constituants immédiats des propositions tels qu'ils apparaissent dans le texte, nous pouvons constater que le nombre d'organisations différentes autour du verbe est assez important ( 27 organisations pour 92 occurrences). En effet, dans les injonctions, les compléments circonstanciels jouant un rôle important, on retrouve différentes configurations où nous avons un ou plusieurs circonstants. Dans notre corpus, le nombre de ces compléments varie de 1 à 3 . Cependant, malgré cette diversité, on constate que finalement, un très petit nombre de ces structures sont utilisées fréquemment. Si nous pouvons relever des occurrences comme :

(25) Au robot culinaire ou au mélangeur, moudre finement la moitié des amandes. (Recette)

(26) S'ils sont sales, nettoyer la fenêtre des émetteurs de lumière et des capteurs avec une petite brosse. (Manuel)

les configurations les plus récurrentes sont les plus simples, dans le sens où elles ne contiennent qu'un nombre restreint de constituants, avec le verbe, l'objet et éventuellement un circonstant, qui a le plus souvent la forme d'un SP :

(27) Contrôler l'équipement nécessaire à la mission de secours. (Référentiel)

(28) Plonger l'électrode dans la solution de pH inconnu. (Manuel)

Les résultats de ce relevé de base des constituants présents dans la proposition indiquent aussi que les configurations les plus variées sont observées dans les recettes. En effet, dans ce type de texte, où la précision est recherchée, et où la façon de procéder, le moyen, ou encore la durée, sont essentiels à la réalisation, les circonstants ont une place importante.

Concernant la structure canonique des verbes relevés, nous comptons un nombre réduit de configurations par rapport au relevé des constituants. Les verbes utilisés dans notre corpus ont majoritairement une construction transitive directe $[\mathrm{V}+\mathrm{SN}]$. Au niveau du nombre de configurations différentes, comme pour la colonne «Constit», on constate que seules deux configurations comptent une occurrence pertinente sur 13 structurations relevées ([V + SN], 59,3\% et [V + SN prép SN], 27,5\%).

Lorsque nous considérons la structure argumentale du verbe, nous comptons 9 structurations, dont trois sont pertinentes $([\mathrm{V}+\mathrm{SN}]$, $[\mathrm{V}+\mathrm{SN}+\mathrm{SPC}]$, [V]). Une fois de plus, les plus fréquentes étant les plus concises. On retrouve autour du verbe un complément direct, et éventuellement un circonstant.

Ces différentes analyses nous montrent que dans le texte injonctif, malgré des phrases en apparence longues, le nombre de constituants présents est très réduit. On constate aussi que la nature du texte joue un certain rôle dans l'organisation syntaxique. Dans la recette, définie comme une « formule indiquant les proportions des substances qui entrent dans certains mélanges et la façon de confectionner les mets » $(T L F i)$, la manière de procéder et la durée sont importantes et on trouve de nombreux circonstants (manière, temps, moyen, lieu...). Dans le manuel d'entretien et de maintenance que nous avons consulté, les consignes concernent une grande diversité d'appareils, et il se veut une aide complémentaire au manuel fourni par le fabricant de l'équipement en question. Ceci pourrait expliquer que les phrases présentent une organisation moins «riche» que dans les recettes. Enfin, dans le référentiel de compétences, on trouve très peu de circonstants puisque pour chaque compétence que l'apprenant devra maîtriser, des consignes supplémentaires seront données dans la partie dédiée, avec plus de précisions.

L'analyse du comportement des circonstants indique que les CC privilégient la position postP, peu importe le nombre de $\mathrm{CC}$ dans la proposition. Nous avons aussi relevé que les circonstances exprimées le 
plus fréquemment étaient différentes selon le texte en question. Le test d'effacement montre que dans le texte injonctif, si les circonstants peuvent être supprimés, cette suppression n'est pas sans conséquences sur le sens de l'énoncé, et n'est donc pas souhaitable même dans le cas de circonstants non arguments.

L'utilisation de l'infinitif dans ce type de texte permet des concisions non négligeables qui permettent de rester précis. La possibilité d'utiliser un verbe en emploi absolu, alors que ce verbe n'admet normalement pas cette construction en est une parfaite illustration.

\section{Conclusion}

Nous avons présenté, dans la première partie de notre étude, les principales caractéristiques du texte injonctif selon différents aspects : morphosyntaxe, sémantique, pragmatique, analyse du discours. Nous nous sommes intéressée plus particulièrement à l'utilisation de l'infinitif dans ces textes. Nous partons de l'idée que dans cet emploi, l'infinitif est une forme verbale à part entière, avec des spécificités qui lui sont propres, comme c'est aussi le cas pour l'impératif. L'analyse du sujet de l'injonction dans les textes avec l'infinitif oblige à interpréter ce sujet comme étant de type humain. En effet, cette forme n'existe qu'à l'écrit en français contemporain, et les verbes de notre corpus se trouvent dans des configurations où d'une part, ils constituent des prédicats actionnels, et d'autre part, ils ont un sens en contexte qui ne permet pas l'attribution d'un sujet non-humain :

(29) À l'aide d'une cuillère de bois, incorporer les brisures de chocolat et le reste des amandes. (Recette)

(30)Contrôler l'équipement nécessaire à la mission de secours. (Référentiel)

(31) Régler l'échelle de lecture. (Manuel)

La deuxième partie de notre travail s'est consacrée à une étude de cas sur corpus. Nous avons relevé des occurrences dans trois types de textes : des recettes de cuisine, des consignes provenant d'un manuel d'entretien et de maintenance des équipements de laboratoire et des consignes d'un référentiel de compétences des secouristes. Nous avons voulu voir si la nature du texte avait une grande incidence sur la structuration syntaxique. Avec la recette, nous nous rapprochons du procédural, et on note l'importance des circonstanciels :

(32) Au robot culinaire ou au mélangeur, moudre finement la moitié des amandes. (Recette)

(33) Déposer les biscuits sur des grilles. (Recette)

(34)Faire chauffer l'huile de tournesol dans un grand wok préchauffé. (Recette)

Dans le manuel d'entretien, qui s'adresse à des professionnels, on retrouve aussi cette forte présence des circonstants, bien qu'ils soient moins nombreux que dans les recettes, dans la mesure où le manuel particulier que nous avons utilisé est complémentaire aux manuels de fabricants :

(35) Brancher l'appareil sur une prise de courant de voltage approprié. (Manuel)

(36) Régler l'échelle de lecture. (Manuel)

Enfin, dans le référentiel de compétences pour des secouristes de niveau 1, ouvrage destiné à un plus large public, on retrouve des structurations plus simples, avec moins de circonstants :

(37) Examiner la victime. (Référentiel) 
(38) Surveiller la ou les victimes dans l'attente des secours. (Référentiel)

En relevant les occurrences comptant au moins un complément circonstanciel, on en compte 24 dans la catégorie « recette », 20 dans le manuel et seulement 6 pour le référentiel.

De manière générale, nous avons pu constater que, malgré quelques spécificités liées à la nature du texte et au public visé, le texte injonctif s'organise de la même manière autour de l'infinitif. On privilégie des structurations concises et l'infinitif permet souvent des tournures qui ne seraient pas possibles à la forme finie, permettant ainsi d'éviter les ambiguïtés. Nous avons aussi pu constater que le nombre de circonstants dans la proposition est restreinte et que leur comportement est fortement contraint par la nature du texte, ainsi que le démontrent les tests d'effacement et de déplacement.

\section{Références du corpus}

Direction de la défense et de la sécurité civiles. (2007). Référentiel national de compétences de sécurité civile relatif aux «Premiers secours en équipe de niveau 1 », $2^{\mathrm{e}}$ édition. Paris.

Organisation mondiale de la Santé. (2008). Manuel d'entretien et de maintenance des appareils de laboratoire. $2^{\mathrm{e}}$ édition. Genève : Éditions de l'OMS.

Davies, S. (2003). Cuisine au wok. Bath : Parragon Books Ltd.

Collectif. (2011). Nos 200 meilleurs desserts et biscuits. Montréal : Les Éditions Transcontinentales.

\section{Références bibliographiques}

ADAM, J.-M. (2001a). Entre conseil et consignes : Les genres de l'incitation à l'action. Pratiques : théorie, pratique, pédagogie (111-112), pp. 7-38.

ADAM, J.-M. (2001b). Types de textes ou genres de discours ? Comment classer les textes qui disent de et comment faire ? Langages (141), pp. 10-27.

ADAM, J.-M. (2004). Linguistique textuelle : Des genres de discours aux textes. Paris : Nathan.

ADAM, J.-M. (2005). Les textes, types et prototypes : récit, description, argumentation et dialogue (éd. 2). Paris : A. Colin, Éd.

BENAYOUN, J.-M. (2003). Sujet $\varnothing$, pacte référentiel et thème. Dans J.-M. MERLE (Éd.), Le sujet (pp. 173-182). Paris : Ophrys.

BRACOPS, M. (2005). Introduction à la pragmatique : les théories fondatrices. Bruxelles : De Boeck.

COLLIN, C. (2006). Le sujet dans l'injonction. Rennes : Presses universitaires de Rennes.

FLOREA, L. S., FUCHS, C., \& MÉLANIE-BECQUET, F. (2010). Dictionnaire des verbes du français actuel. Constructions, emplois, synonymes. Paris : Éditions Ophrys.

GOSSELIN, L. (2010). Les modalités en français. La validation des représentations. Amsterdam: Rodopi.

GROSS, G. Compléments d'objets et compléments circonstanciels. [en ligne] http://www.ruslang.ru/doc/melchuk_festschrift2012/Gross.pdf

HAN, C.-h. (1999). Deontic Modality, Lexical Aspect and the Semantics of Imperatives. Linguistics in morning calm, 4, pp. 479-495. 
KERLEROUX, F. (1996). La coupure invisible: études de syntaxe et de morphologie. Villeneuve d'Ascq : Presses Universitaires du Septentrion.

HEURLEY, L. (2001). Compréhension et utilisation de textes procéduraux : l'effet de l'ordre de mention des informations. Revue française de linguistique appliquée, 6(2), pp. 29-46.

KHODABOCUS, N. (2012). L'infinitif dans le recettes de cuisine. Dans C. DESPIERRES, \& M. KRAZEM, Quand les genres de discours provoquent la grammaire... et réciproquement. (pp. 115-125). Limoges : Lambert-Lucas.

KHODABOCUS, N. (2014). L'infinitif : quelle catégorie ? SHS Web of Conferences - 4e Cogrès Mondial de Linguistique, 8, pp. 2249-2263.

LE QUERLER, N. (1996). Typologie des modalités. Caen : Presses universiatires de Caen.

RIEGEL, M. (2005). À propos du statut verbal de l'infinitif et des formes de son auxiliation. Dans F. LAMBERT, \& H. NØLKE, La syntaxe au coeur de la grammaire (pp. 287-295). Rennes : Presses universitaires de Rennes.

RIEGEL, M., PELLAT, J.-C., \& RIOUL, R. (2009). Grammaire méthodique du français (éd. 7 revue et augmentée). Paris : PUF.

TURNER, N. (2000). Étude contrastive de l'infinitif en français et en anglais. Paris : Éditions Ophrys.

VIKNER, C. (1980). L’infinitif et le syntagme infinitif. Revue Romane, (15-2), pp. 252-291.

\footnotetext{
${ }^{1}$ Nous remercions les relecteurs de cet article pour leurs commentaires pertinents qui nous ont permis d'améliorer ce travail. Nous remercions aussi J. Goes pour sa relecture attentive et ses précieux conseils.

${ }^{2}$ Gosselin, $2010: 361$.

${ }^{3}$ Conseils, Mousse exfoliante démaquillante aux 3 thés Yves Rocher@.

${ }^{4}$ Mode d'emploi, Gel rasage Williams (C) expert gel hydrant.

${ }^{5}$ Les exemples sont ceux de Gosselin (2010).

${ }^{6}$ Nous renvoyons à Khodabocus (2014) pour des explications détaillées sur la catégorisation de l'infinitif, que nous ne pouvons pas développer ici pour des contraintes évidentes d'espace.

${ }^{7}$ Dans [V + SN prép SN], [prép] peut renvoyer à «à » entre autres prépositions. Lorsque le dictionnaire utilise «prép » c'est que le verbe admet plusieurs prépositions. Les données n'indiquent pas que [V + SN prép SN] et [V + $\mathrm{SN}$ à $\mathrm{SN}$ ] s'excluent mutuellement. Lorsque «à » est précisé en revanche, c'est qu'il n'y a que cette préposition qui puisse être utilisée.

${ }^{8}$ Dans le Dictionnaire des verbes du français actuel, les entrées précisent les prépositions pour les constructions n'admettant qu'un nombre très limité de prépositions. La notation [prép] est utilisée lorsque l'éventail de possibilités est plus large. Par exemple, en considérant les verbes de notre corpus, pour incorporer, on trouve :

- $\mathrm{V}+\mathrm{SN}-\mathrm{L}$ 'armée incorpore de jeunes recrues

- $\quad \mathrm{V}+\mathrm{SN}$ à $\mathrm{SN}-\sim$ du beurre à la farine ; les immigrés à la population; un pays à une fédération

- $\quad \mathrm{V}+\mathrm{SN}$ dans $\mathrm{SN}-\sim$ un passage dans un texte

et pour déposer, on trouve :

- $\mathrm{SN}+\mathrm{V}-\mathrm{Ce}$ vin dépose

- $\quad \mathrm{V}+\mathrm{SN}-\sim$ un brevet d'invention ; le bilan; une plainte $[\ldots]$

- $\quad \mathrm{V}+\mathrm{SN}$ prép SN - des fleurs sur une tombe ; un paquet chez Paul ; qqn à la gare ; son argent à la banque

- V contrelen faveur de SN - contrel en faveur de l'accusé
}

${ }^{9}$ Dans les différents textes, les structures les plus fréquentes correspondent à la structure canonique. Nous rappelons que (SP) renvoie à tous les circonstants arguments ou pas. Or, les données indiquent que dans la majorité des occurrences, ce SP correspond à un argument. 